\title{
A purity theorem for the Witt group
}

\author{
Manuel Ojanguren and Ivan Panin
}

\section{Introduction}

We briefly review the definitions of quadratic spaces and Witt groups. A very detailed exposition of these topics may be found in $[\mathbf{8}]$ and in $[\mathbf{9}]$.

Let $X$ be a scheme such that 2 is invertible in $\Gamma\left(\mathcal{O}_{X}\right)$. A quadratic space over $X$ is a pair $\mathbf{q}=(\mathcal{E}, q)$ consisting of a locally free coherent sheaf (we also say "vector bundle") $\mathcal{E}$ and a symmetric isomorphism $q: \mathcal{E} \rightarrow \mathcal{E}^{*}=\operatorname{Hom}_{\mathcal{O}_{X}}\left(\mathcal{E}, \mathcal{O}_{X}\right)$ : this means that, after identifying $\mathcal{E}$ with $\mathcal{E}^{* *}$ in the usual way, it satisfies $q=q^{*}$.

An isometry $\varphi: \mathbf{q} \rightarrow \mathbf{q}^{\prime}$ is an isomorphism $\varphi: \mathcal{E} \rightarrow \mathcal{E}^{\prime}$ such that the square

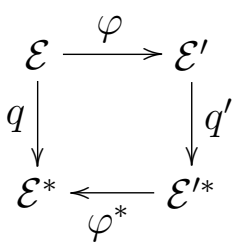

commutes.

The orthogonal sum of $\mathbf{q}$ and $\mathbf{q}^{\prime}$ is the space $\mathbf{q} \perp \mathbf{q}^{\prime}=\left(\mathcal{E} \oplus \mathcal{E}^{\prime}, q \oplus q^{\prime}\right)$.

Let $\mathbf{q}=(\mathcal{E}, q)$ be a quadratic space over $X$ and $\mathcal{F}$ a subsheaf of $\mathcal{E}$. The orthogonal $\mathcal{F}^{\perp}$ of $\mathcal{F}$ is the kernel of $q \circ i^{*}$, where $i$ denotes the inclusion of $\mathcal{F}$ into $\mathcal{E}$.

A subbundle $\mathcal{L}$ of $\mathcal{E}$ is a sublagrangian of $\mathbf{q}$ if $\mathcal{L} \subseteq \mathcal{L}^{\perp}$, and it is a lagrangian if $\mathcal{L}=\mathcal{L}^{\perp}$. Note that lagrangians and sublagrangians are subbundles, i.e. locally direct factors, not just subsheaves. A space $\mathbf{q}=(\mathcal{E}, q)$ is said to be metabolic if it has a lagrangian.

Let $\mathrm{GW}(X)$ denote the Grothendieck group of quadratic spaces over $X$ with respect to the orthogonal sum. Let $M$ be the subgroup of $\mathrm{GW}(X)$ generated by metabolic spaces. The Witt group of $X$ is the quotient $\mathrm{W}(X)=\mathrm{GW}(X) / M$. If $f: X \rightarrow Y$ is a map of schemes and $\mathbf{q}=(\mathcal{E}, q)$ is space over $Y$, the pair $f^{*} \mathbf{q}=\left(f^{*} \mathcal{E}, f^{*} q\right)$ is a quadratic space over $X$. It is easily seen that $f^{*}$ respects orthogonal sums and maps metabolic spaces to metabolic spaces, thus $f$ induces a group homomorphism $\mathrm{W}(f): \mathrm{W}(Y) \rightarrow \mathrm{W}(X)$ and $\mathrm{W}$ turns out to be a contravariant functor from the category of schemes to the category of abelian groups.

If $X=\operatorname{Spec}(A)$ is affine, a quadratic space over $X$ is the same as a pair $(P, q)$ consisting of a finitely projective $A$-module $P$ and an $A$-linear isomorphism $q: P \rightarrow P^{*}$ such that $q=q^{*}$. In this case a space $(P, q)$ is metabolic if and only if it is isometric to a space of the form $\left(L \oplus L^{*},\left(\begin{array}{ll}0 & 1 \\ 1 & 0\end{array}\right)\right)$.

For an affine scheme $X=\operatorname{Spec}(A)$ we denote $\mathrm{W}(X)$ by $\mathrm{W}(A)$.

Let now $X$ be an integral scheme and $K=k(X)$ its field of rational functions. By the functoriality of $\mathrm{W}$ there is a canonical map $\mathrm{W}(X) \rightarrow \mathrm{W}(K)$ and, for every point $x \in X$, a canonical map $\mathrm{W}\left(\mathcal{O}_{X, x}\right) \rightarrow \mathrm{W}(K)$. We say that an element $\xi \in \mathrm{W}(K)$ is unramified 
at $x$ if $\xi$ is in the image of $\mathrm{W}\left(\mathcal{O}_{X, x}\right)$. We say that an element $\xi \in \mathrm{W}(K)$ is unramified (over $X$ ) if it is unramified at every height one point $x \in X$. We say that purity holds for $X$ if every unramified element of $\mathrm{W}(K)$ belongs to the image of $\mathrm{W}(X)$ in $\mathrm{W}(K)$.

Purity is known to hold for every regular integral noetherian scheme of dimension at most two [3] and for every regular integral noetherian affine scheme of dimension three [14].

The main result of this paper is the following purity theorem $(\S 7)$.

Theorem A. Purity holds for any regular local ring containing a field of characteristic $\neq 2$.

Theorem A will be deduced from the same statement for essentially smooth local algebras over a field, using a well-known result of Dorin Popescu.

Further, using essentially the same methods, we prove ( $§ 8)$

Theorem B. Let $A$ be a regular local ring containing a field of characteristic $\neq 2$ and $K$ the field of fractions of $A$. Let $f$ be a regular parameter of $A$. The natural homomorphism $\mathrm{W}\left(A_{f}\right) \rightarrow \mathrm{W}(K)$ is injective.

From this, using a result of Piotr Jaworski for 2-dimensional regular rings, we deduce $(\S 9)$

Theorem C. Let $A$ be a regular local ring containing a field of characteristic $\neq 2$ and $f$ a regular parameter of $A$. There is a short exact sequence

$$
0 \longrightarrow \mathrm{W}(A) \longrightarrow \mathrm{W}\left(A_{f}\right) \stackrel{\delta}{\longrightarrow} \mathrm{W}(A / A f) \longrightarrow 0
$$

where $\delta$ is the restriction to $\mathrm{W}\left(A_{f}\right)$ of the second residue homomorphism $\partial_{f}$ at the height one prime $\mathfrak{p}=A f$.

Let $A((t))=A[[t]]_{t}$ be the ring of formal Laurent series over $A$. As a special case of Theorem $\mathrm{C}$ we can formulate $(\S 9)$

Theorem D. Let $A$ be a regular local ring containing a field of characteristic $\neq 2$. There exists a split short exact sequence

$$
0 \rightarrow \mathrm{W}(A) \rightarrow \mathrm{W}(A((t))) \rightarrow \mathrm{W}(A) \rightarrow 0
$$

Remark. The method used for proving purity for an essentially smooth local $k$-algebra $A$ also yields a new proof of the injectivity of $\mathrm{W}(A)$ into the Witt group $\mathrm{W}(K)$ of its field of fractions. Since this result is well-known and not very difficult (see for instance $[\mathbf{1 3}]$ ) we use it whenever it is convenient, without proving it again.

Our proof has been inspired by Vladimir Voevodsky's work [19] and makes essential use of a non-degenerate trace form for finite extensions of smooth algebras, which was discovered by Leonhard Euler in a special case. We recall its definition and main properties in $\S \S 2$ and 3 .

Acknowledgements. We heartly thank Slava Kopeiko for asking the right question at the right moment, Andrei Suslin for critically following an oral exposition of our results and Jean-Louis Colliot-Thélène for his comments on the first draft of this paper. We are grateful to the SNSF, the INTAS, the RFFI, the Fondation Herbette and the Fondation Chuard-Schmid for financial support. 


\section{The Euler trace}

Let $k$ be any field and $A \hookrightarrow B$ a finite extension of smooth, purely $d$-dimensional $k$ algebras. Let $\Omega_{A}$ and $\Omega_{B}$ be the modules of Kähler differentials of $A$ and $B$ over $k$ and let $\Omega_{B / A}$ be the module of relative differentials of $B$ over $A$. Let $\omega_{A}=\bigwedge^{d} \Omega_{A}$, $\omega_{B}=\bigwedge^{d} \Omega_{B}$.

Proposition 2.1. There exists an isomorphism of $B$-modules $\omega_{B} \simeq \operatorname{Hom}_{A}\left(B, \omega_{A}\right)$.

Proof. Let $R$ be the polynomial algebra $A\left[X_{1}, \ldots, X_{n}\right]$ and $\rho: R \rightarrow B$ a surjective homomorphism of $A$-algebras. Let $I=\operatorname{ker}(\rho)$. Since $B$ is a local complete intersection over $A$, by Lemma 4.4 of [17] there exists an isomorphism of $B$-modules

$$
\operatorname{Hom}_{A}(B, A) \simeq \bigwedge^{n}\left(\operatorname{Hom}_{B}\left(I / I^{2}, B\right)\right)
$$

On the other hand, from the canonical exact sequence of projective $B$-modules (see $[\mathbf{1}]$, VII, Theorem 5.8)

$$
0 \rightarrow I / I^{2} \rightarrow B \otimes_{R} \Omega_{R} \rightarrow \Omega_{B} \rightarrow 0
$$

we deduce, taking maximal exterior powers, that

$$
\omega_{B} \otimes_{B} \bigwedge^{n}\left(I / I^{2}\right) \simeq B \otimes_{A} \omega_{A}
$$

From $(\dagger)$ we get, using the fact that $I / I^{2}$ is a finitely generated projective $B$-module,

$$
\omega_{B} \simeq\left(B \otimes_{A} \omega_{A}\right) \otimes_{B} \operatorname{Hom}_{B}\left(\bigwedge^{n}\left(I / I^{2}\right), B\right) \simeq\left(B \otimes_{A} \omega_{A}\right) \otimes_{B} \bigwedge^{n}\left(\operatorname{Hom}_{B}\left(I / I^{2}, B\right)\right)
$$

and then, from $(*)$,

$$
\left(B \otimes_{A} \omega_{A}\right) \otimes_{B} \bigwedge^{n}\left(\operatorname{Hom}_{B}\left(I / I^{2}, B\right)\right) \simeq \omega_{A} \otimes_{A} \operatorname{Hom}_{A}(B, A) \simeq \operatorname{Hom}_{A}\left(B, \omega_{A}\right) .
$$

Corollary 2.2. If $\omega_{A}$ and $\omega_{B}$ are trivial there exists an isomorphism of $B$-modules

$$
\lambda: B \simeq \operatorname{Hom}_{A}(B, A) .
$$

The isomorphism $\lambda$ induces an $A$-linear map

$$
\mathfrak{e}: B \rightarrow A
$$

defined by $\mathfrak{e}(x)=\lambda(1)(x)$. We call it an Euler trace, because Euler discovered a special case of it (see [5] and also [16], Chap. III). Conversely, from $\mathfrak{e}$ we get back $\lambda$ as $\lambda(x)(y)=$ $\mathfrak{e}(x y)$.

In the next proposition we record, without proof, a few obvious properties of $\mathfrak{e}$ and $\lambda$.

Proposition 2.3. Let $B$ be a finite locally free $A$-algebra and $\mathfrak{e}: B \rightarrow A$ an $A$-linear map such that the bilinear map

$$
\lambda: B \rightarrow \operatorname{Hom}_{A}(B, A) \text { given by } \lambda(x)(y)=\mathfrak{e}(x y)
$$

is an isomorphism. Then, for every $A \rightarrow A^{\prime}$, we have an $A^{\prime}$-linear map

$$
\mathfrak{e}^{\prime}=\mathfrak{e} \otimes_{A} A^{\prime}: B^{\prime}=B \otimes_{A} A^{\prime} \rightarrow A^{\prime}
$$

such that the associated $\lambda^{\prime}: B^{\prime} \rightarrow \operatorname{Hom}_{A^{\prime}}\left(B^{\prime}, A^{\prime}\right)$ is an isomorphism of $B^{\prime}$-modules. If $B=B_{1} \times B_{2}, \lambda$ decomposes as $\lambda_{1} \times \lambda_{2}$, where $\lambda_{i}: B_{i} \rightarrow \operatorname{Hom}_{A}\left(B_{i}, A\right)$ is the map associated to $\left.\mathfrak{e}\right|_{B_{i}}$. In particular, if $B=B_{1} \times A$ the map $\lambda_{2}: A \rightarrow A$ is the multiplication by a unit of $A$. 


\section{Traces and quadratic spaces}

Let $A \hookrightarrow B$ be a finite flat extension of commutative rings. Let $\mathfrak{e}: B \rightarrow A$ be an $A$ linear map such that the associated $\lambda: B \rightarrow \operatorname{Hom}_{A}(B, A)$ is an isomorphism. To every quadratic space $\mathbf{q}=(P, q)$ over $B$ we associate the bilinear form $\operatorname{Tr}^{\mathfrak{e}}(\mathbf{q})=\left(P_{A}, \mathfrak{e} \circ q\right)$, where $P_{A}$ denotes $P$ considered as an $A$-module. This bilinear form is in fact a quadratic space and it is easy to check (see $[\mathbf{9}], \mathrm{I}, \S 7$ ) that $\operatorname{Tr}$ has the following properties:

(1) $\operatorname{Tr}^{\mathfrak{e}}\left(\mathbf{q} \perp \mathbf{q}^{\prime}\right)=\operatorname{Tr}^{\mathfrak{e}}(\mathbf{q}) \perp \operatorname{Tr}^{\mathfrak{e}}\left(\mathbf{q}^{\prime}\right)$.

(2) If $\mathbf{q}$ is hyperbolic, $\operatorname{Tr}^{\mathfrak{e}}(\mathbf{q})$ is hyperbolic.

(3) For any homomorphism of commutative rings $A \rightarrow A^{\prime}$ we have

$$
\operatorname{Tr}^{\mathfrak{e}^{\prime}}\left(\mathbf{q} \otimes_{A} A^{\prime}\right)=\operatorname{Tr}^{\mathfrak{e}}(\mathbf{q}) \otimes_{A} A^{\prime}
$$

where $\mathfrak{e}^{\prime}=\mathfrak{e} \otimes_{A} A^{\prime}$.

(4) If, as at the end of $\S 2, B=B_{1} \times B_{2}$ and $\mathfrak{e}_{i}=\left.\mathfrak{e}\right|_{B_{i}}$,

$$
\operatorname{Tr}^{\mathfrak{e}}(\mathbf{q})=\operatorname{Tr}^{\mathfrak{e}_{1}}\left(\mathbf{q}_{1}\right) \perp \operatorname{Tr}^{\mathfrak{e}_{2}}\left(\mathbf{q}_{2}\right)
$$

where $\mathbf{q}_{i}=\mathbf{q} \otimes_{B} B_{i}$.

(5) If, as in (4), $B=B_{1} \times B_{2}$ but $B_{2}=A$, then $\mathfrak{e}_{2}$ is the multiplication by a unit $u \in A^{*}$ and thus, for any quadratic space $\mathbf{q}$,

$$
\operatorname{Tr}^{\mathfrak{e}_{2}}\left(\mathbf{q}_{2}\right)=u \cdot \mathbf{q}_{2}
$$

If $f: A \rightarrow A^{\prime}$ is a ring homomorphism and $B^{\prime}=B \otimes_{A} A^{\prime}$, clearly $B^{\prime}=B_{1}^{\prime} \times B_{2}^{\prime}$ with $B_{2}^{\prime}=A^{\prime}$ and $\mathfrak{e}_{2}^{\prime}$ is the multiplication by $f(u)$.

(6) Suppose that the map $f: A \rightarrow A^{\prime}$ considered in (5) has a section $s: A^{\prime} \rightarrow A$ and that $B \otimes_{A} A^{\prime}=B^{\prime}=B_{1}^{\prime} \times B_{2}^{\prime}$ with $B_{2}^{\prime}=A^{\prime}$. Then, by (5), $\mathfrak{e}_{2}^{\prime}$ is the multiplication by a unit $u^{\prime}$ of $A^{\prime}$. Replacing $\mathfrak{e}$ by $s\left(u^{\prime}\right)^{-1} \mathfrak{e}$ we get a new Euler map $\mathfrak{e}: B \rightarrow A$ for which $\mathfrak{e}_{2}^{\prime}=\operatorname{id}_{A^{\prime}}$ and for any ring homomorphism $A^{\prime} \rightarrow A^{\prime \prime}$ we have $B^{\prime \prime}=B_{1}^{\prime \prime} \times B_{2}^{\prime \prime}$ with $B_{2}^{\prime \prime}=A^{\prime \prime}$ and $\mathfrak{e}_{2}^{\prime \prime}=\operatorname{id}_{A^{\prime \prime}}$. Thus, for any quadratic space $\mathbf{q}^{\prime \prime}$ over $B^{\prime \prime}$

$$
\operatorname{Tr}_{2}^{\mathfrak{e}_{2}^{\prime \prime}}\left(\mathbf{q}_{2}^{\prime \prime}\right)=\mathbf{q}_{2}^{\prime \prime}
$$

(7) The linear map $\mathfrak{e}: B \rightarrow A$ induces a homomorphism of Witt groups

$$
\operatorname{Tr}^{\mathfrak{e}}: \mathrm{W}(B) \rightarrow \mathrm{W}(A)
$$

(8) If $B$ is of the form $A[t] /(f)=A[\tau]$, where $f$ is a monic polynomial of odd degree and $\tau$ the class of $t$, we can define an Euler map by

$$
\mathfrak{e}\left(\tau^{i}\right)= \begin{cases}0 & \text { if } i<n-1 \\ 1 & \text { if } i=n-1\end{cases}
$$

In this case a direct computation shows that the composite homomorphism

$$
\mathrm{W}(A) \rightarrow \mathrm{W}(B) \rightarrow \mathrm{W}(A)
$$

is the identity of $\mathrm{W}(A)$. 


\section{Reduction of purity to infinite base fields}

Let $\mathbb{F}$ be a finite field of odd characteristic $p$ and $A$ a local, essentially smooth $\mathbb{F}$-algebra with maximal ideal $\mathfrak{m}$. Suppose that purity holds for essentially smooth local algebras over any infinite field $k$. Let $K$ be the field of fractions of $A$ and $\xi$ an unramified element of $\mathrm{W}(K)$. Let $p^{m}$ be the cardinality of $A / \mathfrak{m}$ and $s$ an odd integer greater than 2 and prime to $m$. For any $i$ let $k_{i}$ be the field (in some fixed algebraic closure of $\mathbb{F}$ ) of degree $s^{i}$ over $\mathbb{F}$. Let $k$ be the union of all $k_{i}$. Since $k \otimes_{\mathbb{F}}(A / \mathfrak{m})$ is still a field, $B=k \otimes_{\mathbb{F}} A$ is a local, essentially smooth algebra over the infinite field $k$. Let $L=k \otimes_{\mathbb{F}} K$ be its field of fractions. The image $\xi_{L}$ of $\xi$ in $\mathrm{W}(L)$ is unramified. In fact, let $\mathfrak{q}$ be a height one prime of $B$ and $\mathfrak{p}=A \cap \mathfrak{q}$. By assumption $\xi \in \mathrm{W}\left(A_{\mathfrak{p}}\right)$ and since $A_{\mathfrak{p}} \rightarrow L$ factors through $B_{\mathfrak{q}}$ the class $\xi_{L}$ is in $\mathrm{W}\left(B_{\mathfrak{q}}\right)$ for every $\mathfrak{q}$. Since purity holds for $B, \xi_{L} \in \mathrm{W}(B)$. We can now find a finite subfield $\mathbb{F}^{\prime}$ of $k$ and, for $A^{\prime}=\mathbb{F}^{\prime} \otimes_{\mathbb{F}} A$ a $\xi^{\prime} \in \mathrm{W}\left(A^{\prime}\right)$ which maps to $\xi_{L}$. Let $K^{\prime}$ be the field of fractions of $A^{\prime}$. Further enlarging $\mathbb{F}^{\prime}$ we may assume that the images of $\xi$ and $\xi^{\prime}$ in $\mathrm{W}\left(K^{\prime}\right)$ coincide. Consider now the diagram

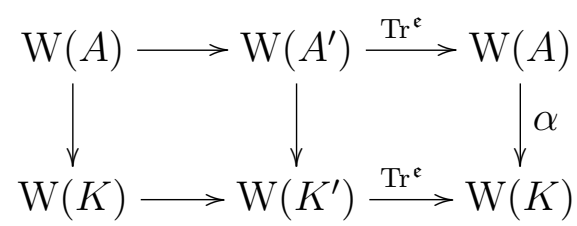

where $\mathfrak{e}$ has been chosen as in $\S 3(8)$. Since the composition of the horizontal maps is the identity, we have $\alpha \circ \operatorname{Tr}\left(\xi^{\prime}\right)=\xi$ in $\mathrm{W}(K)$. Thus $\xi$ is indeed in the image of $\mathrm{W}(A)$.

\section{The geometric presentation lemma}

We state and prove a lemma that will play a crucial role in the sequel. In geometrical disguise it sounds like this:

Lemma 5.1. Let $A$ be a local ring of a smooth variety over an infinite field $k$. Let $U=\operatorname{Spec}(A)$ and let $u$ be the closed point of $U$. Let $p: \mathcal{X} \rightarrow U$ be an affine $U$-scheme, essentially smooth over $k$. Let $f$ be a regular element of $k[\mathcal{X}]$ such that $k[\mathcal{X}] /(f)$ is finite over $A$. We denote by $\mathcal{X}_{f}$ the principal open set defined by $f \neq 0$. Assume that there exists a finite surjective morphism $\mathcal{X} \rightarrow U \times \mathbb{A}_{k}^{1}$ of $U$-schemes and that there exists a section $\Delta: U \rightarrow \mathcal{X}$ of $p$ such that $p$ is smooth along $\Delta(U)$.

Then there exists a finite surjective morphism

$$
\pi: \mathcal{X} \rightarrow U \times \mathbb{A}_{k}^{1}
$$

of $U$-schemes with the following properties:

(a) $\pi^{-1}(U \times\{1\})$ is in $\mathcal{X}_{f}$.

(b) $\pi^{-1}(U \times\{0\})=\Delta(U) \amalg \mathcal{D}$, where $\mathcal{D} \subset \mathcal{X}_{f}$.

Clearly the statement above is equivalent to the following, purely algebraic one.

Lemma 5.2. Let $A$ be a local essentially smooth algebra over an infinite field $k, \mathfrak{m}$ its maximal ideal and $R$ an essentially smooth $k$-algebra, which is finite over the polynomial algebra $A[t]$. Suppose that $\epsilon: R \rightarrow A$ is an $A$-augmentation and let $I=\operatorname{ker}(\epsilon)$. Assume that $R$ is smooth over $A$ at every prime containing $I$. Given $f \in R$ such that $R / R f$ is finite over $A$ we can find an $s \in R$ such that 
(1) $R$ is finite over $A[s]$.

(2) $R / R s=R / I \times R / J$ for some ideal $J$ of $R$.

(3) $J+R f=R$.

(4) $R(s-1)+R f=R$.

Proof. Replacing $t$ by $t-\epsilon(t)$ we may assume that $t \in I$. We denote by "bar" the reduction modulo $\mathfrak{m}$. By the assumptions made on $R$ the quotient $\bar{R}$ is smooth over $\bar{A}$ at its maximal ideal $\bar{I}$. Choose an $\alpha \in R$ such that $\bar{\alpha}$ is a local parameter of the localization $\bar{R}_{\bar{I}}$ of $\bar{R}$ at $\bar{I}$. By the chinese remainders' theorem we may assume that $\bar{\alpha}$ does not vanish at the zeros of $\bar{f}$ different from $\bar{I}$. Without changing $\bar{\alpha}$ we may replace $\alpha$ by $\alpha-\epsilon(\alpha)$ and assume that $\alpha \in I$. Since $R$ is integral over $A[t]$ there exists a relation of integral dependence

$$
\alpha^{n}+p_{1}(t) \alpha^{n-1}+\ldots+p_{n}(t)=0 .
$$

For any $r \in k^{*}$ and any $N$ larger than the degree of each $p_{i}(t)$, putting $s=\alpha-r t^{N}$ we see that from the equation above that $t$ is integral over $A[s]$. Hence $R$, which is integral over $A[t]$, is integral over $A[s]$. Clearly $s \in I$. To insure that $\bar{s}$ is also a local parameter of $\bar{R}_{\bar{I}}$ it suffices to take $n \geq 2$. By assumption $R$ and $A[s]$ are both regular and since $R$ is finite over $A[s], R$ is locally free over $A[s]$ (see for instance Corollary 18.17 of [4]) and hence $R / R s$ is free over $A$. Since $\bar{s}$ is a local parameter of $\bar{R}_{\bar{I}}, \bar{R} / \bar{s} \bar{R}$ is étale over $\bar{A}$ at the augmentation ideal $\bar{I}$ and so we can find a $g \notin I+\mathfrak{m} R$ such that $(R / R s)_{g}$ is étale over $A$. By the next sublemma $R / R s$ splits as in (2).

Sublemma 5.3. Let $B$ be a commutative ring, $\gamma: B \rightarrow C$ a finite commutative $B$ algebra and $\lambda: C \rightarrow B$ an augmentation with augmentation ideal $I$. Let $h \in C$ be such that

(a) $C_{h}$ is étale over $B$.

(b) $\lambda(h)$ is invertible in $B$.

Then $C$ splits as $C / I \times C / J$ for some ideal $J$ of $C$.

Proof. Since $B \rightarrow C_{h}$ is étale and the composite map

$$
B \stackrel{\gamma}{\longrightarrow} C_{h} \stackrel{\lambda}{\longrightarrow} B
$$

is the identity of $B$, by Prop. 4.7 of $[\mathbf{1}], C_{h} \rightarrow B$ is étale. But $C \rightarrow C_{h}$ is étale, hence $\lambda$ : $C \rightarrow B$ is étale and in particular it induces an open morphism $\lambda^{*}: \operatorname{Spec}(B) \rightarrow \operatorname{Spec}(C)$. Its image $\lambda^{*}(\operatorname{Spec}(B)=\operatorname{Spec}(C / I)$ is therefore open and since it is also closed, $C$ splits as claimed.

To finish the proof of Lemma 5.2 we still have to choose $r \in k^{*}$ so that conditions (3) and (4) are satisfied. Since $R / R f$ is semilocal, there are only finitely many maximal ideals of $R$ containing $f$. We denote by $\mathfrak{m}_{1}, \ldots, \mathfrak{m}_{p}$ those which, in case $f \in I+\mathfrak{m} R$, are different from $I+\mathfrak{m} R$. Recalling that $\alpha$ was chosen outside $\mathfrak{m}_{1} \cup \ldots \cup \mathfrak{m}_{p}$, we have $s \notin \mathfrak{m}_{1} \cup \ldots \cup \mathfrak{m}_{p}$ for almost any choice of $r \in k^{*}$. To see that condition (3) is satisfied it suffices to show that $J \nsubseteq \mathfrak{m}_{i}$ for $1 \leq i \leq p$ and that $J \nsubseteq \mathfrak{m} R+I$. The first assertion is clear because $s \in J \backslash \mathfrak{m}_{i}$ for $1 \leq i \leq p$. For the second one note that, since $R / R s=R / I \times R / J$, we have $I+J=R$ and therefore $J \nsubseteq \mathfrak{m} R+I$. It remains to satisfy (4). Since $R / R f$ is semilocal there exists a $\lambda \in k$ such that $s-\lambda$ is invertible in $R / R f$. Without perturbing conditions (1), (2) and (3) we may replace $s$ by $\frac{1}{\lambda} s$ and thus satisfy (4) as well. 


\section{A commutative diagram for relative curves}

Lemma 6.1. With the notation and the hypotheses of Lemma 5.2, let $U=\operatorname{Spec}(A)$ and $\mathcal{X}=\operatorname{Spec}(R)$. Let $p: \mathcal{X} \rightarrow U$ be the structural morphism and $\Delta: U \rightarrow \mathcal{X}$ the morphism corresponding to the augmentation $\epsilon: R \rightarrow A$. Let $\mathcal{Z} \subset \mathcal{X}$ be a closed set of codimension at least 2 , contained in vanishing locus of $f$. Suppose that $\omega_{\mathcal{X} / k}$ is trivial. There exists a nonzero element $g \in A$ such that $\mathcal{X}_{g} \subseteq \mathcal{X} \backslash \mathcal{Z}$ and for any such $g$ there exists a commutative diagram

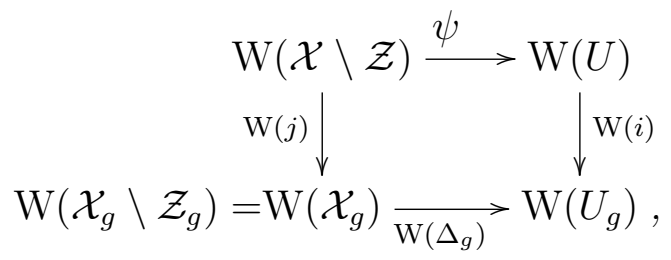

where $i: U_{g} \rightarrow U$ and $j: \mathcal{X}_{g} \rightarrow \mathcal{X} \backslash \mathcal{Z}$ are the inclusions.

Proof. By Lemma 5.2 there exists an element $s \in R$ satisfying the conditions (1) to (4). The evaluation in $s$ defines a finite surjective morphism $\pi: \mathcal{X} \rightarrow U \times \mathbb{A}_{k}^{1}$ of $U$ schemes such that $\pi^{-1}(U \times\{0\})=\Delta(U) \amalg \mathcal{D}_{0}$ with $\mathcal{D}_{0} \subset \mathcal{X}_{f}$. Since $\omega_{U \times \mathbb{A}_{k}^{1} / k}$ is obviously trivial and $\omega_{\mathcal{X} / k}$ is trivial by assumption, we can use Corollary 2.2 to find an Euler trace $\mathfrak{e}: B \rightarrow A[t]$ such that the associated map $\lambda: B \rightarrow \operatorname{Hom}_{A[t]}(B, A[t])$ is an isomorphism. We can then choose a trace map $\operatorname{Tr}: \mathrm{W}(\mathcal{X}) \rightarrow \mathrm{W}\left(U \times \mathbb{A}_{k}^{1}\right)$ as in $\S 3$. Restricting $\operatorname{Tr}$ to $\mathrm{W}\left(\pi^{-1}(U \times\{0\})\right.$ yields a homomorphism $\mathrm{W}\left(\pi^{-1}(U \times\{0\}) \rightarrow \mathrm{W}(U \times\{0\})\right.$. Since the natural embedding $A \hookrightarrow A[t]$ is a section of the evaluation at $t=0$, by (6) of $\S 3$ we may choose the Euler trace $\mathfrak{e}: B \rightarrow A[t]$ such that $\left.\operatorname{Tr}\right|_{\mathrm{W}(\Delta(U))}=\mathrm{W}(\Delta)$.

Having fixed $\mathfrak{e}$ and $\operatorname{Tr}$ in this way, restricting $\mathfrak{e}$ to $\mathcal{D}_{i}, i=1,2$, we get trace maps $\operatorname{Tr}_{i}: \mathrm{W}\left(\mathcal{D}_{i}\right) \rightarrow \mathrm{W}(U)$. Let $\varphi_{i}: \mathcal{D}_{i} \rightarrow \mathcal{X} \backslash \mathcal{Z}$ be the inclusion. We put

$$
\psi=\operatorname{Tr}_{1} \circ \mathrm{W}\left(\varphi_{1}\right)-\operatorname{Tr}_{0} \circ \mathrm{W}\left(\varphi_{0}\right) .
$$

Since $\mathcal{Z}$ is of codimension $\geq 2$ in $\mathcal{X}$ and $\pi: \mathcal{X} \rightarrow U \times \mathbb{A}_{k}^{1}$ is finite, the image of $\mathcal{Z}$ in $U$ under the structural map is contained in the vanishing locus of some non zero $g \in A$. Making now the base change of $\mathfrak{e}$ by means of the inclusion $i: U_{g} \hookrightarrow U$ we get $\mathfrak{e}_{g}$ and $\operatorname{Tr}_{g}$ such that we still have $\left.\operatorname{Tr}_{g}\right|_{\mathrm{W}\left(\Delta\left(U_{g}\right)\right)}=\mathrm{W}\left(\Delta_{g}\right)$ (see (6) of $\S 3$ ). Further restricting $\mathfrak{e}_{g}$ to $\mathcal{D}_{i g}, i=1,2$, we get trace maps $\operatorname{Tr}_{i g}: \mathrm{W}\left(\mathcal{D}_{i g}\right) \rightarrow \mathrm{W}\left(U_{g}\right)$. Let $\varphi_{i g}: \mathcal{D}_{i g} \rightarrow \mathcal{X}_{g} \backslash \mathcal{Z}_{g}=\mathcal{X}_{g}$, $i=1,2$, be the inclusions. We put

$$
\psi_{g}=\operatorname{Tr}_{1 g} \circ \mathrm{W}\left(\varphi_{1 g}\right)-\operatorname{Tr}_{0 g} \circ \mathrm{W}\left(\varphi_{0_{g}}\right) .
$$

Clearly properties (3) and (4) of $\S 3$ imply the relation $\mathrm{W}(i) \circ \psi=\psi_{g} \circ \mathrm{W}(j)$. Thus, to complete the proof of the lemma, it suffices to check the relation $\psi_{g}=\mathrm{W}\left(\Delta_{g}\right)$. For this take any $\xi$ in $\mathrm{W}\left(\mathcal{X}_{g}\right)$ and write a chain of relations

$\left.\operatorname{Tr}_{g}(\xi)\right|_{U_{g} \times\{1\}}-\left.\operatorname{Tr}_{g}(\xi)\right|_{U_{g} \times\{0\}}=\operatorname{Tr}_{1 g}\left(\left.\xi\right|_{\mathcal{D}_{1 g}}\right)-\operatorname{Tr}_{0 g}\left(\left.\xi\right|_{\mathcal{D}_{0 g}}\right)-\operatorname{Tr}_{g}\left(\left.\xi\right|_{\Delta\left(U_{g}\right)}\right)=\psi_{g}(\xi)-\mathrm{W}\left(\Delta_{g}\right)(\xi)$.

A well-known theorem of Max Karoubi (see [9], VII, §4) asserts that for any affine $k$ scheme $S$ the canonical homomorphism $\mathrm{W}(S) \rightarrow \mathrm{W}\left(S \times \mathbb{A}_{k}^{1}\right)$ is an isomorphism, and therefore the left hand side of the relation above is zero. This proves the relation $\psi_{g}=$ $\mathrm{W}\left(\Delta_{g}\right)$, whence the commutativity of the diagram. 


\section{Purity}

Theorem 7.1. Let $A$ be a local, essentially smooth algebra over an infinite field $k$ and let $K$ be its field of fractions. Every unramified element of $\mathrm{W}(K)$ belongs to $\mathrm{W}(A)$.

Proof. Let $U=\operatorname{Spec}(A)$ and let $\xi$ be an unramified element of $\mathrm{W}(K)$. By assumption there exist a smooth $d$-dimensional $k$-algebra $R=k\left[t_{1}, \ldots, t_{n}\right]$ and a prime ideal $\mathfrak{p}$ of $R$ such that $A=R_{\mathfrak{p}}$. We first reduce the proof to the case in which $\mathfrak{p}$ is maximal. To do this, choose a maximal ideal $\mathfrak{m}$ containing $\mathfrak{p}$. Since $k$ is infinite, by a standard general position argument we can find $d$ algebraically independent elements $X_{1}, \ldots, X_{d}$ such that $R$ is finite over $k\left[X_{1}, \ldots, X_{d}\right]$ and étale at $\mathfrak{m}$. After a linear change of coordinates we may assume that $R / \mathfrak{p}$ is finite over $B=k\left[X_{1}, \ldots, X_{m}\right]$, where $m$ is the dimension of $R / \mathfrak{p}$. Clearly $R$ is smooth over $B$ at $\mathfrak{m}$ and thus, for some $h \in R \backslash \mathfrak{m}$, the localization $R_{h}$ is smooth over $B$. Let $S$ be the set of nonzero elements of $B, k^{\prime}=S^{-1} B$ the field of fractions of $B$ and $R^{\prime}=S^{-1} R_{h}$. The prime ideal $\mathfrak{p}^{\prime}=S^{-1} \mathfrak{p}_{h}$ is maximal in $R^{\prime}$, the $k^{\prime}$-algebra $R^{\prime}$ is smooth and $A=R_{\mathfrak{p}^{\prime}}^{\prime}$.

From now on we assume that $A=\mathcal{O}_{X, x}$ is the local ring of a closed point $x$ of a smooth $d$-dimensional affine variety $X$ over $k$.

Replacing $X$ by a sufficiently small affine neighbourhood of $x$ we may assume that $\omega_{X / k}$ is trivial. By Proposition 2.4 of [3] we may assume that $\xi$ is defined on the complement of a closed set $Z$ of codimension at least 2 in $X$. Let $f \neq 0$ be a regular function on $X$ which vanishes on a closed set $Y$ containing $Z$. By Quillen's trick (see [15], Lemma 5.12) we can find a morphism $q: X \rightarrow \mathbb{A}_{k}^{d-1}$ with the following properties:

(1) $q$ is smooth at $x$.

(2) $\left.q\right|_{Y}: Y \rightarrow \mathbb{A}_{k}^{d-1}$ is finite.

(3) $q$ factors as

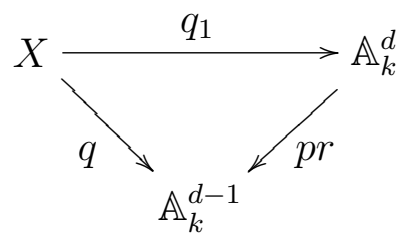

with $q_{1}$ finite and surjective.

Consider the cartesian square

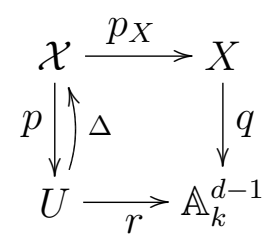

where $U=\operatorname{Spec}\left(\mathcal{O}_{X, x}\right), r=\left.q\right|_{U}, \mathcal{X}=U \times_{\mathbb{A}_{k}^{d-1}} X, p$ is the first projection and $\Delta: U \rightarrow \mathcal{X}$ the diagonal. Denote again by $f$ the composition of $f$ with $p_{X}$.

Since $r$ is smooth, $p_{X}$ is also smooth and since $X$ is smooth over $k$, so is $\mathcal{X}$. By base change, condition (3) implies that $\mathcal{X}$ is an affine relative curve over $U$. Since $U$ is local and $q$ is smooth at $x, p$ is smooth along $\Delta(U)$. From (3), by base change via 
$r: U \rightarrow \mathbb{A}_{k}^{d-1}$, we get a commutative triangle

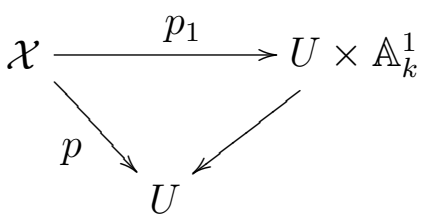

with $p_{1}$ finite. Again by the same base change we see that $k[\mathcal{X}] /(f)$ is finite over $A$. Thus all the hypotheses of Lemma 5.1 are satisfied and we can find a $U$-morphism $\pi$ : $\mathcal{X} \rightarrow U \times \mathbb{A}_{k}^{1}$ satisfying conditions (a) and (b).

We further claim that $\omega_{\mathcal{X}}$ is trivial. To see this observe that

$$
\omega_{\mathcal{X} / k} \simeq p_{X}^{*}\left(\omega_{X / k}\right) \otimes_{\mathcal{O}_{\mathcal{X}}} \omega_{\mathcal{X} / X}
$$

and that $\omega_{\mathcal{X} / X} \simeq p^{*} \omega_{U / \mathbb{A}_{k}^{d-1}}$. Since $U$ is essentially smooth over $\mathbb{A}_{k}^{d-1}, \omega_{U / \mathbb{A}_{k}^{d-1}}$ is locally free of rank one, hence trivial because $U$ is local. Thus $p^{*} \omega_{U / \mathbb{A}_{k}^{d-1}}$ is trivial and, since $\omega_{X / k}$ is trivial by assumption, we conclude that $\omega_{\mathcal{X} / k}$ is trivial.

We can now apply Lemma 6.1 with $\mathcal{Z}=U \times_{\mathbb{A}_{k}^{d-1}} Z \subset \mathcal{X}$. We define $\eta=\psi\left(\mathrm{W}\left(p_{X}\right)(\xi)\right)$ and claim that $\eta$ is an extension of $\xi$ to $U$. In fact, choosing $g \in A$ as in 6.1 and denoting by $i: U_{g} \rightarrow U, i^{\prime}: U_{g} \rightarrow X \backslash Z$ and $j: \mathcal{X}_{g} \rightarrow \mathcal{X} \backslash \mathcal{Z}$ the inclusions, we have

$$
\mathrm{W}(i) \eta=\mathrm{W}(i) \circ \psi \circ \mathrm{W}\left(p_{X}\right) \xi=\mathrm{W}\left(\Delta_{g}\right) \circ \mathrm{W}(j) \circ \mathrm{W}\left(p_{X}\right) \xi=\mathrm{W}\left(p_{X} \circ j \circ \Delta_{g}\right) \xi=\mathrm{W}\left(i^{\prime}\right) \xi
$$

This completes the proof of Theorem 7.1.

To prove Theorem A we now recall a celebrated result of Dorin Popescu (see [10], [11] and $[\mathbf{1 2}]$ or $[\mathbf{2}]$ or, for a self-contained proof, [18]).

Le $k$ be a field and $R$ a local $k$-algebra. We say that $R$ is geometrically regular if $k^{\prime} \otimes_{k} R$ is regular for any finite extension $k^{\prime}$ of $k$. A ring homomorphism $A \rightarrow R$ is called geometrically regular if it is flat and for each prime ideal $\mathfrak{q}$ of $R$ lying over $\mathfrak{p}$, $R_{\mathfrak{q}} / \mathfrak{p} R_{\mathfrak{q}}=k(\mathfrak{p}) \otimes_{A} R_{\mathfrak{q}}$ is geometrically regular over $k(\mathfrak{p})=A_{\mathfrak{p}} / \mathfrak{p}_{\mathfrak{p}}$.

Observe that any regular local ring containing a field $k$ is geometrically regular over the prime field of $k$.

Popescu's theorem. A homomorphism $A \rightarrow R$ of noetherian rings is geometrically regular if and only if $R$ is a filtered direct limit of smooth $A$-algebras.

Proof of Theorem $A$. Let $R$ be a regular local ring containing a field. Let $k$ be the prime field of $R$. By Popescu's theorem $R=\lim _{\rightarrow} A_{\alpha}$, where the $A_{\alpha}$ 's are smooth $k$-algebras. We first observe that we may replace the direct system of the $A_{\alpha}$ 's by a system of essentially smooth local $k$-algebras. In fact, if $\mathfrak{m}$ is the maximal ideal of $R$, we can replace each $A_{\alpha}$ by $\left(A_{\alpha}\right)_{\mathfrak{p}_{\alpha}}$, where $\mathfrak{p}_{\alpha}=\mathfrak{m} \cap A_{\alpha}$. Note that in this case the canonical morphisms $\varphi_{\alpha}: A_{\alpha} \rightarrow R$ are local and every $A_{\alpha}$ is a regular local ring, in particular a factorial ring.

Let now $L$ be the field of fractions of $R$ and, for each $\alpha$ let $K_{\alpha}$ be the field of fractions of $A_{\alpha}$. Let $\xi$ be an unramified element of $\mathrm{W}(L)$. We may represent $\xi$ by a diagonal matrix $q=\operatorname{diag}\left(r_{1}, \ldots, r_{n}\right)$ with $r_{1}, \ldots, r_{n}$ in $R$. Let $\Sigma$ be the (finite) set of height one primes of $R$ which divide at least one of the $r_{i}$. For every $\mathfrak{p} \in \Sigma$ we can find a matrix 
$\sigma(\mathfrak{p}) \in G l_{n}(L)$ that transforms $q$ into a diagonal form $\operatorname{diag}\left(u_{1}(\mathfrak{p}), \ldots, u_{n}(\mathfrak{p})\right)$ with every $u_{i}(\mathfrak{p}) \in R \backslash \mathfrak{p}$. Clearing denominators we may assume that $\sigma(\mathfrak{p}) \in M_{n}(R)$ and that

$$
\sigma(\mathfrak{p})^{T} q \sigma(\mathfrak{p})=\operatorname{diag}\left(u_{1}(\mathfrak{p}), \ldots, u_{n}(\mathfrak{p})\right)(d(\mathfrak{p}))^{2}
$$

for some $d(\mathfrak{p}) \in R$. We can now choose an index $\alpha$ such that, for every $\mathfrak{p} \in \Sigma, A_{\alpha}$ contains preimages $\tilde{r}_{1}, \ldots, \tilde{r}_{n}, \tilde{u}_{1}(\mathfrak{p}), \ldots, \tilde{u}_{n}(\mathfrak{p}), \tilde{d}(\mathfrak{p})$ and $\tilde{\sigma}_{i j}(\mathfrak{p})$ of the elements $r_{1}, \ldots, r_{n}$, $u_{1}(\mathfrak{p}), \ldots, u_{n}(\mathfrak{p}), d(\mathfrak{p})$ and of the coefficients $\sigma_{i j}(\mathfrak{p})$ of $\sigma(\mathfrak{p})$. Having chosen these preimages consider the relations

$$
\tilde{\sigma}(\mathfrak{p})^{T} \tilde{q} \tilde{\sigma}(\mathfrak{p})=\operatorname{diag}\left(\tilde{u}_{1}(\mathfrak{p}), \ldots, \tilde{u}_{n}(\mathfrak{p})\right)(\tilde{d}(\mathfrak{p}))^{2}
$$

where $\tilde{q}=\operatorname{diag}\left(\tilde{r}_{1}, \ldots, \tilde{r}_{n}\right)$ and $\tilde{\sigma}(\mathfrak{p})$ is the matrix $\left(\tilde{\sigma}_{i j}(\mathfrak{p})\right)$. Since they hold over $R$, we may assume, after replacing $\alpha$ by some larger index, that they hold over $A_{\alpha}$. We claim that the class of $\tilde{q}$ (which we still denote by $\tilde{q}$ ) is an unramified element of $\mathrm{W}\left(K_{\alpha}\right)$. To show this suppose that $\tilde{q}$ is ramified at a height one prime ideal $p A_{\alpha}$. Then $p$ divides some $\tilde{r}_{i}$. Any height one prime $\mathfrak{p}$ of $R$ containing $p R$ also contains $r_{i}$ and thus belongs to $\Sigma$. Since $u_{i}(\mathfrak{p}) \in R \backslash \mathfrak{p}$ we have $\tilde{u}_{i}(\mathfrak{p}) \in A_{\alpha} \backslash p A_{\alpha}$ and thus the relation $(\star)$ shows that $\tilde{q}$ is unramified at $p A_{\alpha}$. By purity for $A_{\alpha}$ there exists a $\xi_{\alpha} \in \mathrm{W}\left(A_{\alpha}\right)$ that coincides with $\tilde{q}$ in $\mathrm{W}\left(K_{\alpha}\right)$. The ideal $\mathfrak{r}=\operatorname{ker}\left(\varphi_{\alpha}\right)$ is prime and does not contain any $\tilde{r}_{i}$. Hence $\tilde{q}$ is a quadratic space over the essentially smooth local algebra $B_{\alpha}=\left(A_{\alpha}\right)_{\mathfrak{r}}$. Since $\tilde{q}$ and $\xi_{\alpha}$ coincide in $\mathrm{W}\left(K_{\alpha}\right)$ they already coincide in $\mathrm{W}\left(B_{\alpha}\right)$ because $\mathrm{W}\left(B_{\alpha}\right) \rightarrow \mathrm{W}\left(K_{\alpha}\right)$ is injective. The commutative diagram of ring homomorphisms

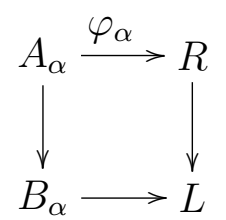

shows that $\mathrm{W}\left(\varphi_{\alpha}\right)\left(\xi_{\alpha}\right)=q$ in $\mathrm{W}(L)$. This proves that $q$ is indeed in $\mathrm{W}(R)$.

\section{An injectivity theorem}

If $A$ is a regular ring of dimension greater than 3 and $K$ its field of fractions, the canonical homomorphism $\mathrm{W}(A) \rightarrow \mathrm{W}(K)$ need not be injective. In this section we prove the following injectivity result, from which we shall deduce Theorem $\mathrm{C}$.

Theorem 8.1. Let $A$ be a local, essentially smooth algebra over an infinite field $k$ of characteristic $\neq 2$. Let $K$ be the field of fractions of $A$ and $f$ a regular parameter of $A$. The canonical homomorphism $\mathrm{W}\left(A_{f}\right) \rightarrow \mathrm{W}(K)$ is injective.

The proof of this theorem is similar to that of Theorem 7.1. As we did there, we assume, without loss of generality, that $A$ is the local ring of a closed point $x$ of a smooth affine variety $X$. If $A$ is 1-dimensional $A_{f}=K$ and there is nothing to prove, so we assume that $A$ is at least 2-dimensional. We need the following variant of Quillen's trick.

Lemma 8.2. Let $X$ be an irreducible affine smooth variety over an infinite field $k$ and $x$ a closed point of $X$. Let $A$ be the local ring of $x, f \in k[X]$ a regular function on $X$ which is a regular parameter of $A$ and $g \in k[X], g$ prime to $f$. Denote by $Y$ the vanishing 
locus of $f$ and by $Z$ the vanishing locus of $g$. There exists a morphism $q: X \rightarrow \mathbb{A}_{k}^{d-1}$ with the following properties:

(1) $q$ is smooth at $x$.

(2) $\left.q\right|_{Y \cap Z}: Y \cap Z \rightarrow \mathbb{A}_{k}^{d-1}$ is finite.

(3) $q$ factors as

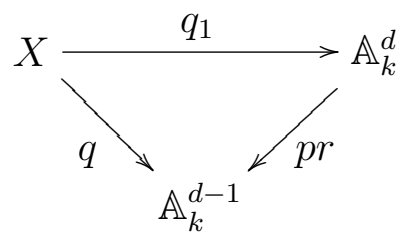

with $q_{1}$ finite and surjective.

(4) $q(Y)=\{0\} \times \mathbb{A}_{k}^{d-2}$

(5) $q^{-1}\left(\{0\} \times \mathbb{A}_{k}^{d-2}\right)=Y \cup Y^{\prime}$ for some closed set $Y^{\prime} \subset X$ which avoids $x$.

We first recall an auxiliary result, which has been proved in slightly different versions by several authors.

Lemma 8.3. Under the assumptions of Lemma 8.2 there exists a morphism $q_{2}: X \rightarrow \mathbb{A}_{k}^{d}$ such that

(i) $q_{2}$ is finite.

(ii) $q_{2}$ is étale at $x$.

(iii) $k(x)=k\left(q_{2}(x)\right)$.

(iv) $Y \cap q_{2}^{-1}\left(q_{2}(x)\right)=\{x\}$.

Proof. Suppose that $X$ is a closed set of $\mathbb{A}_{k}^{N} \subset \mathbb{P}^{N}$ and let $\bar{X}$ be its closure in $\mathbb{P}^{N}$. To prove Lemma 8.3 we will take for $q_{2}$ the projection from a suitable linear subspace $L$ at infinity. Let $\bar{k}$ be an algebraic closure of $k$ and $\varphi: \bar{k} \otimes_{k} X \rightarrow X$ the canonical projection. Then $\varphi^{-1}(x)$ is a finite set of closed points $\left\{x_{1}, \ldots, x_{n}\right\}$ of $\bar{k} \otimes_{k} X$. Choose an $N-d$-1-dimensional linear subspace $L$ in $\mathbb{P}^{N} \backslash \mathbb{A}_{k}^{N}$ with the following properties:

(a) $L$ is defined over $k$.

(b) $L$ does not intersect $\bar{k} \otimes_{k} \bar{X}$.

(c) $L$ does not intersect the tangent planes of $\bar{k} \otimes_{k} \bar{X}$ at $x_{1}, \ldots, x_{n}$.

(d) For $i \neq j$ we have $q_{2}\left(x_{i}\right) \neq q_{2}\left(x_{j}\right)$.

(e) $L$ does not intersect the closures of the cones with vertices $x_{1}, \ldots, x_{n}$ and base $\bar{k} \otimes_{k} Y$.

Dimension considerations show the existence of infinitely many such linear spaces. Condition (a) insures that $q_{2}$ is defined over $k$. Condition (b) insures that $q_{2}: X \rightarrow \mathbb{A}_{k}^{d}$ is finite. Condition (c) insures that $q_{2}$ is étale at $x$. Since the group $\operatorname{Aut}_{k}(\bar{k})$ acts transitively on $\left\{x_{1}, \ldots, x_{n}\right\}$, by condition (d) it acts transitively on $\left\{q_{2}\left(x_{1}\right), \ldots, q_{2}\left(x_{n}\right)\right\}$ as well. This shows that the separability degree of $k\left(q_{2}(x)\right)$ over $k$ is the same as that of $k(x)$. But $q_{2}$ is étale at $x$, hence the extension $k(x) / k\left(q_{2}(x)\right)$, being separable, must be of degree one. Thus condition (iii) is satisfied. Finally, condition (iv) follows from (e).

Proof of Lemma 8.2. We choose $q_{2}$ as in the previous lemma. We put $B=k\left[\mathbb{A}_{k}^{d}\right]$ and $C=k[X]$. The map $q_{2}$ induces an inclusion $\iota: B \hookrightarrow C$ and $C$ is a finite $B$-module. The images of the closed subschemes $Y=\{f=0\}$ and $Z=\{g=0\}$ of $X$ are two closed closed subschemes of $\mathbb{A}_{k}^{d}$ defined, respectively, by $f_{0}=0$ and $g_{0}=0$ for some $f_{0}, g_{0} \in k\left[\mathbb{A}_{k}^{d}\right]$. The inclusion $\iota$ induces a finite map $B / B f_{0} \rightarrow C / C f$. Let $\mathfrak{m}$ be the maximal ideal of $B$ corresponding to the closed point $q_{2}(x)$. Since $x$ is the unique closed 
point of $Y$ lying over $q_{2}(x)$, the localization $(C / C f)_{\mathfrak{m}}=B_{\mathfrak{m}} \otimes_{B}(C / C f)$ is local and finite over $\left(B / B f_{0}\right)_{m}$. By condition (iii) these two local rings have the same residue field, hence by Nakayama's lemma they are isomorphic. This shows in particular that $f_{0}$ is a regular parameter of $B$ at $q_{2}(x)$. On the other hand, since $C$ is étale over $B$ at $x$, $f_{0}$ is also a regular parameter of $C$ at $x$.

We now have two polynomials $f_{0}$ and $g_{0}$ in $B=k\left[X_{1}, \ldots, X_{d}\right]$ which we may assume monic in, say, $X_{1}$. The map $k\left[Y_{1}, \ldots, Y_{d}\right] \rightarrow k\left[X_{1}, \ldots, X_{d}\right]$ defined by $Y_{1} \mapsto f_{0}$ and $Y_{i} \mapsto X_{i}$ for $i \neq 1$ induces a finite morphism $q_{3}: \mathbb{A}_{k}^{d} \rightarrow \mathbb{A}_{k}^{d}$. Composing $q_{2}$ with $q_{3}$ we obtain a finite map $q_{1}=q_{3} \circ q_{2}: X \rightarrow \mathbb{A}_{k}^{d}$. This map is smooth at $x$ because $q_{2}$ is étale at $x$ and $f_{0}$ is a regular parameter at $q_{2}(x)$. It maps $Y$ onto the hyperplane $Y_{1}=0$ and $Z$ onto some closed set $\left\{g_{1}=0\right\}$. Since $q_{1}$ is a local-étale isomorphism at $x, Y_{1}$ does not divide $g_{1}$. Thus $q_{1}(Y \cap Z)$ is a proper closed subset of the hyperplane $Y_{1}=0$. We may therefore assume, after a linear change of coordinates involving only $Y_{2}, \ldots, Y_{d}$, that the projection $p r$ onto $Y_{2}=0$ is finite on $q_{1}(Y \cap Z)$. We now take $q=p r \circ q_{1}$.

Since $q^{-1}\left(\{0\} \times \mathbb{A}_{k}^{d-2}\right)$ is smooth at $x$, it contains only one component - namely $Y$ that passes through $x$, whence (5).

Proof of Theorem 8.1. Let $\xi$ be an element in the kernel of $\mathrm{W}\left(A_{f}\right) \rightarrow \mathrm{W}(K)$. There is a $g \in A$, which we may suppose prime to $f$, such that $\xi \in \operatorname{ker}\left(\mathrm{W}\left(A_{f}\right) \rightarrow \mathrm{W}\left(A_{f g}\right)\right) \mathrm{We}$ may represent $\xi$ by a quadratic space $\mathbf{q}$ defined over $A_{f}$ which becomes hyperbolic over $A_{f g}$. Patching q over $\operatorname{Spec}\left(A_{f}\right)$ with a suitable hyperbolic space over $\operatorname{Spec}\left(A_{g}\right)$ we get a space over the complement of the closed set $W=Y \cap Z$, where $Y=\{f=0\}$ and $Z=\{g=0\}$. Applying Lemma 8.2 we get a map $q: X \rightarrow \mathbb{A}_{k}^{d-1}$ satisfying properties (1) to (5). Let $h \in k[X]$ be an element which vanishes identically on $W$ and such that $q$ is finite on the closed subscheme defined by $\{h=0\}$. As in the proof of Theorem 7.1, but with $h$ instead of $f$ and $W$ instead of $Z$, we get a commutative square

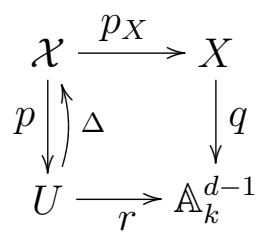

where $U=\operatorname{Spec}\left(\mathcal{O}_{X, x}\right), r=\left.q\right|_{U}, \mathcal{X}=U \times_{\mathbb{A}_{k}^{d-1}} X, p$ is the first projection and $\Delta$ : $U \rightarrow \mathcal{X}$ the diagonal. We denote again by $h$ the composition of $h$ with $p_{X}$ and we put $\mathcal{W}=U \times_{\mathbb{A}_{k}^{d-1}} W$. As in the proof of 7.1 , we assume that $X$ has been so chosen that $\omega_{X}$ is trivial.

Applying the geometric presentation lemma we find a map $\pi: \mathcal{X} \rightarrow U \times \mathbb{A}_{k}^{1}$ of $U$-schemes such that $\pi^{-1}(U \times\{1\})=\mathcal{D}_{1}$ is in $\mathcal{X}_{h}$ and $\pi^{-1}(U \times\{0\})=\Delta(U) \amalg \mathcal{D}_{0}$, where $\mathcal{D}_{0} \subset \mathcal{X}_{h}$. Put for simplicity $s=Y_{2}$. By condition (5) we have $\mathcal{W} \subset \mathcal{X} \backslash \mathcal{X}_{s}$ and hence, by Lemma 6.1 , there exists a commutative square

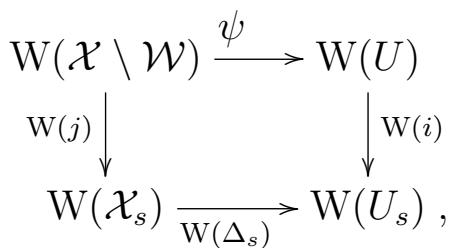


where $i: U_{s} \rightarrow U$ and $j: \mathcal{X}_{s} \rightarrow \mathcal{X} \backslash \mathcal{W}$ are the inclusions. Repeating the argument of the proof of Theorem 7.1, we define $\eta=\psi\left(\mathrm{W}\left(p_{X}\right)(\xi)\right) \in \mathrm{W}(A)$ and get $\eta_{s}=\xi_{s}$. By condition (5), $A_{s}=A_{f}$ and since $\mathrm{W}(A) \rightarrow \mathrm{W}(K)$ is injective and $\xi$ vanishes on $\mathrm{W}(K)$ we get $\eta=0$. This shows that $\xi=0$ as well.

Proof of Theorem B. We first extend Theorem 8.1 to the case of an infinite base field. This is even simpler than for Theorem A: we find a sufficiently large odd degree extension $\mathbb{F}^{\prime}$ of the finite base field $\mathbb{F}$ such that $A^{\prime}=\mathbb{F}^{\prime} \otimes_{\mathbb{F}} A$ is still a local ring and $\xi_{\mathbb{F}^{\prime}}=0$ in $\mathrm{W}\left(A^{\prime}\right)$. Then, choosing $\mathfrak{e}$ as in $\S 3$, (8), we see that $\xi=\operatorname{Tr}^{\mathfrak{e}}\left(\xi_{\mathbb{F}^{\prime}}\right)=0$.

We now prove Theorem B. Let $R$ be a regular local ring containing a field and let $L$ be the field of fractions of $R$. Let $k$ be the prime field of $R$. As in the proof of Theorem A, $R=\lim _{\rightarrow} A_{\alpha}$, where the $A_{\alpha}$ 's are essentially smooth local $k$-algebras. Let $f$ be a regular parameter of $R$ and $\xi$ an element in the kernel of $\mathrm{W}\left(R_{f}\right) \rightarrow \mathrm{W}(L)$. There exists a $g \in R$ such that $\xi$ vanishes in $\mathrm{W}\left(R_{f g}\right)$. For a suitable index $\alpha$ choose lifts $f_{\alpha}$ and $g_{\alpha}$ of $f$ and $g$ in $A_{\alpha}$. We may replace the filtered direct system of the $A_{\alpha}$ by the subsystem of all $A_{\beta}$ with $\beta \geq \alpha$. Clearly we still have $R=\lim _{\beta \geq \alpha} A_{\beta}$. We put, for every $\beta \geq \alpha, f_{\beta}=\varphi_{\beta \alpha}\left(f_{\alpha}\right)$ and $g_{\beta}=\varphi_{\beta \alpha}\left(g_{\alpha}\right)$ where the $\varphi_{\beta \alpha}: A_{\alpha} \rightarrow A_{\beta}$ are the transition homomorphisms. It is easy to see that $\lim _{\beta \geq \alpha}\left(A_{\beta}\right)_{f_{\beta}}=R_{f}$ and $\lim _{\beta \geq \alpha}\left(A_{\beta}\right)_{f_{\beta} g_{\beta}}=R_{f g}$. Since the functor W commutes with filtered direct limits, we have

$$
\lim _{\beta \geq \alpha} \operatorname{ker}\left(\mathrm{W}\left(\left(A_{\beta}\right)_{f_{\beta}}\right) \rightarrow \mathrm{W}\left(\left(A_{\beta}\right)_{f_{\beta} g_{\beta}}\right)\right)=\operatorname{ker}\left(\mathrm{W}\left(R_{f}\right) \rightarrow \mathcal{W}\left(R_{f g}\right)\right)
$$

Since $\varphi_{\beta}: A_{\beta} \rightarrow R$ is local, $f_{\beta}$ is a regular parameter of $A_{\beta}$. Hence the left hand side vanishes and, in particular, $\xi=0$. This proves Theorem B.

\section{A short exact sequence}

Let $B$ be a discrete valuation ring, $\mathfrak{p}=B p$ its maximal ideal and $L$ its field of fractions. Let $v: L^{*} \rightarrow \mathbb{Z}$ be the corresponding valuation of $L$. Recall that there is a homomorphism (which depends on the choice of the local parameter $p$ ) $\partial_{p}: \mathrm{W}(L) \rightarrow \mathrm{W}(B / \mathfrak{p})$ called second residue and defined on rank one forms $\left\langle u p^{m}>\right.$ with $u \in B^{*}$ by

$$
\partial_{p}\left(<u p^{m}>\right)= \begin{cases}0 & \text { if } m \text { is even } \\ <\bar{u}> & \text { if } m \text { is odd }\end{cases}
$$

where $\bar{u}$ is the image of $u$ in $B / \mathfrak{p}$.

This homomorphism fits into the exact sequence

$$
0 \longrightarrow \mathrm{W}(B) \longrightarrow \mathrm{W}(L) \stackrel{\partial_{p}}{\longrightarrow} \mathrm{W}(B / \mathfrak{p}) \longrightarrow 0 \text {. }
$$

Proof of Theorem $C$. We have a commutative diagram

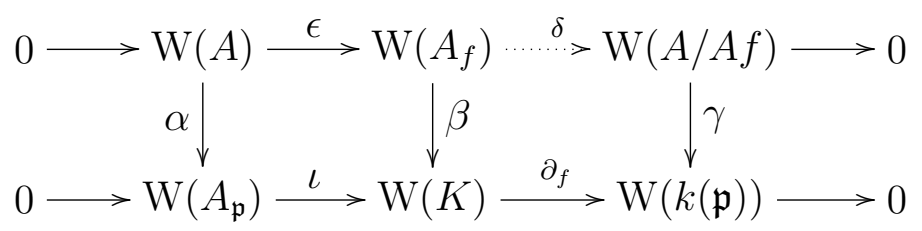

of solid arrows in which the bottom line is exact. We first want to show that

$$
\partial_{f} \circ \beta\left(\mathrm{W}\left(A_{f}\right)\right) \subseteq \mathrm{W}(A / A f)
$$


and then check that the top line is exact.

For the first assertion it suffices to show, by purity, that, for any $\xi \in \mathrm{W}\left(A_{f}\right), \partial_{f} \circ \beta(\xi)$ is unramified over $A / A f$. Let $\mathfrak{q} / A f$ be a prime of height one of $A / A f$. We want to show that $\partial_{f} \circ \beta(\xi)$ is in the image of $\mathrm{W}\left(A_{\mathfrak{q}} / A_{\mathfrak{q}} f\right)$. For this, after replacing $A$ by $A_{\mathfrak{q}}$ in the diagram above, we may assume that $A$ is a local regular ring of dimension 2. But in this case the assertion is precisely Theorem 3 of [7].

Exactness left and right is obvious. Let $\xi$ be an element of $\operatorname{ker}(\delta)$. Since $\beta$ is injective, we may consider $\xi$ as an element of $\mathrm{W}(K)$. From the exactness of the bottom line we see that $\xi$ is in the image of $\mathrm{W}\left(A_{\mathfrak{p}}\right)$. Since it also belongs to $\beta\left(\mathrm{W}\left(A_{f}\right)\right)$, it is unramified and by purity it comes from $\mathrm{W}(A)$.

Proof of Theorem D. Apply Theorem $\mathrm{C}$ to the local ring $A[[t]]$, taking $t$ as regular parameter and using the fact that $\mathrm{W}(A[[t]])=\mathrm{W}(A)$.

\section{References}

1. A. Altman and S. Kleiman: Introduction to Grothendieck duality theory. Lect. Notes in Math. 146, Springer, 1970

2. M. André: Cinq exposés sur la désingularisation (manuscript). École Polytechnique Fédérale de Lausanne, 1991.

3. J.-L. Colliot-Thélène et J.-J. Sansuc: Fibrés quadratiques et composantes connexes réelles. Math. Ann. 244 (1979), 105-134.

4. D. Eisenbud: Commutative Algebra. Graduate Texts in Mathematics 150, Springer, 1994.

5. L. Euler: Theorema arithmeticum eiusque demonstratio. In Leonhardi Euleri Opera Omnia, series I, opera mathematica, volumen VI. Teubner, 1921.

6. F. Fernandez-Carmena: On the injectivity of the map of the Witt group of a scheme into the Witt group of its quotient field. Math. Ann. 277 (1987), 453-481.

7. P. Jaworski: Witt rings of fields of quotients of two-dimensional regular local rings. Math. Z. 211 (1992), 533-546.

8. M. Knebusch: Symmetric and bilinear form over algebraic varieties. In Conference on quadratic forms, Queen's papers in pure and applied mathematics 46, Kingston, Ontario, 1977.

9. M.-A. Knus: Quadratic and Hermitian Forms over Rings. Grundlehren der Math. Wissenschaften 294, Springer, 1991.

10. D. Popescu: General Néron desingularization. Nagoya Math. J. 100 (1985), 97-126.

11. D. Popescu: General Néron desingularization and approximation. Nagoya Math. J. 104 (1986), 85-115.

12. D. Popescu: Letter to the Editor; General Néron desingularization and approximation. Nagoya Math. J. 118 (1990), 45-53.

13. M. Ojanguren: Quadratic forms over regular rings. J. Indian Math. Soc. 44 (1980), $109-116$. 
14. M. Ojanguren, R. Parimala, R. Sridharan and V. Suresh: A purity theorem for the Witt groups of 3-dimensional regular local rings. Proc. London Math. Soc. (to appear).

15. D. Quillen: Higher algebraic $K$-theory I. In Algebraic K-Theory I. Lect. Notes in Math. 341, Springer, 1973.

16. J-P. Serre: Corps locaux. Hermann, Paris, 1962.

17. G. Scheja und U. Storch: Quasi-Frobenius-Algebren und lokal vollständige Durchschnitte. Manuscripta Math. 19 (1976), 75-104.

18. R.G. Swan: Néron-Popescu desingularization. Preprint.

19. V. Voevodsky: Homology of schemes II. Preprint.

August 6, 1997

Manuel Ojanguren, IMA, UNIL, CH-1015 Lausanne, Switzerland

Ivan Panin, LOMI, Fontanka 27, Saint Petersburg 191011, Russia 